\title{
CMS tracker upgrade
}

\section{Duccio Abbaneo* ${ }^{* \dagger}$ \\ CERN}

E-mail: Duccio.Abbaneo@cern.ch

Plans are being made to increase the LHC luminosity above the original nominal value of $10^{34} \mathrm{~cm}^{-2} \mathrm{~s}^{-1}$, eventually achieving an order of magnitude increase at the Super-LHC. CMS needs an upgraded tracking system to maintain adequate performance in the SLHC environment, and to provide tracking information for the level-1 trigger decision. The most relevant requirements and constraints are summarized, along with highlights from some of the R\&D activities.

VERTEX 2009 (18th workshop)

September 13-18 2009

VELUWE, the Netherlands

\footnotetext{
* Speaker.

†n behalf of the CMS Tracker Collaboration
} 


\section{Introduction}

LHC detectors have been designed for a peak luminosity of $10^{34} \mathrm{~cm}^{-2} \mathrm{~s}^{-1}$ and an integrated luminosity of $500 \mathrm{fb}^{-1}$ of $14 \mathrm{TeV}$ p-p collisions. Some physics studies like Higgs couplings or SUSY spectroscopy would profit from even higher statistics, and plans are being developed for a luminosity upgrade of the LHC (Super-LHC or SLHC), eventually delivering a peak luminosity of up to $10^{35} \mathrm{~cm}^{-2} \mathrm{~s}^{-1}$, to achieve an integrated luminosity of $\approx 5000 \mathrm{fb}^{-1}$.

The upgrade plans consist of two main steps: the first involves the integration of the LINAC4 and an upgrade of the collimation system (phase I), while the second implies a completely new injection system (phase II). Phase I would be realized during a winter shutdown, while phase II would imply to stop operations for a full year.

Most of the CMS detector is expected to operate efficiently also at the SLHC, but some parts will require substantial upgrades. In particular the Silicon Tracker will suffer from too high occupancy due to the higher particle density, and degraded performance from the integrated radiation damage. The upgrade of the Tracker is expected to be realized in two steps, matching the accelerators upgrade. The first step will consist of an upgrade of the inner pixel detector, which can be replaced during a Winter shutdown, while the second step will involve the replacement of the whole Tracker, which needs at least one year for installation, connection and commissioning.

The upgrades require major technological developments, and $R \& D$ has begun to address the most important challenges: radiation tolerance of sensors and electronics, distribution of power for both electronics and sensors, removal of heat loads, which may significantly exceed those in the present detector, development of low-power high-performance electronics in advanced technologies. CMS has also identified a novel requirement, which is to provide tracker data to contribute to the level-1 trigger, that must maintain the $100 \mathrm{kHz}$ rate for compatibility with existing sub-detector systems, while increasing the trigger decision latency by only a few $\mu$ s. This paper describes some of the current ideas for developing solutions that could fulfill such requirements, focusing on the phase II upgrade of the full Tracker. The first upgrade of the pixel detector will be only briefly mentioned, since it is covered by other contributions in this conference.

\section{Experience with the current Tracker}

The CMS Tracker consists of about about $200 \mathrm{~m}^{2}$ of silicon sensors with 10 million strip channels plus an inner pixel part with about 66 million channels. A full description of CMS and the Tracker can be found in [1,2]. After about seven years of construction, the Strip Tracker has been installed December 2007, followed by the Pixel Tracker in July 2008. After about 6 month for connecting and commissioning, the Tracker participated in global cosmic data taking with magnetic field in 2008 and 2009 (CRAFT08 and CRAFT09). About 8 million tracks were recorded with the strip tracker and about 85,000 with the pixel detector. $98 \%$ of the strip tracker is working with an efficiency greater than $99 \%$, a signal to noise greater than 25 , and a position resolution of $14-40 \mu \mathrm{m}$ in $\mathrm{r}-\phi$ depending on angle and strip pitch. $99 \%^{1}$ of the pixel detector is operational with an efficiency greater than $95 \%$ (limited only by the random arrival time of the cosmics). The resolution is $20 \mu \mathrm{m}$ in $\mathrm{r}-\phi$ and $30 \mu \mathrm{m}$ in r-z.

\footnotetext{
${ }^{1} 6 \%$ of the forward pixel has been fixed in 2009 . This required a removal - repair and re-insertion of the detector.
} 


\section{Requirements and constraints for the Tracker Upgrade}

Phase I Upgrade: Pixel detector The pixel detector was designed to withstand a fluence of $6 \times 10^{14}$, which corresponds, for the first layer, to a luminosity of $\approx 100 \mathrm{fb}^{-1}$, after which the position resolution starts to degrade. For this reason, and to allow beam-pipe bake-out every two years, the mechanics and the services arrangement of the pixel detector were optimized to allow for a relatively easy access for extraction, maintenance and replacement, which was demonstrated in 2009 with an intervention for the maintenance of the forward part.

With the peak luminosity increasing above $10^{34} \mathrm{~cm}^{-2} \mathrm{~s}^{-1}$, significant dead time will arise in the inner parts of the pixel detector, due to the limited size of on-chip buffers. CMS is planning to replace the current pixel detector with an upgraded system ("phase I upgrade"), featuring improved electronics to minimize dead time, one more pixel layer in both the barrel and the forward part, and a substantial reduction of the passive material in the tracking volume. The new detector, besides ensuring adequate readout efficiency, will provide enhanced tracking performance and robustness for physics analysis.

Phase II Upgrade: the whole tracking system The increased peak and integrated luminosity expected at SLHC translated directly to a need for higher granularity and greater radiation resistance for the new Tracker, in order to maintain the occupancy at the \% level and survive the accumulated fluence. In addition to those requirements driven by the higher luminosity, it would be highly desirable to reduce the material in the Tracking volume, which is at present the limiting factor for the performance of the track reconstruction, and of the electromagnetic energy measurement in CMS.

The design of the new Tracker must take into account important constraints coming from the integration inside CMS. Besides the obvious requirement of fitting inside the same physical envelope, the most significant boundary condition is the necessity to re-use the existing network of services connecting the detector patch panel to the back-end. Tracker cables, pipes and fibers have been laid along complicated paths, interleaved with those of the barrel calorimeters, which will not be upgraded for SLHC. To disentangle and replace those services is not feasible within a oneyear stop. Re-using the services has many important implications for the detector design, namely: (i) the total cross section of conductors for power distribution is fixed, and the power dissipated in the cables is limited by the cable channels thermalization system: the two constraints together translate to a limit in the current that can be delivered to the detector; (ii) flow and pressure for the coolant are limited by the available cross section of cooling pipes and their pressure rating; (iii) the temperature of the coolant has a lower limit due to the insulation in the cable channels, that prevents condensation of water on electrical cables and connectors; (iv) data links have to be fast enough to transmit the whole data volume through the existing optical fiber plant.

Besides maintaining the current tracking performance in the more congested SLHC environment, a novel and most challenging requirement has been identified for the phase II Tracker upgrade, which consists in contributing to the level-1 trigger. The trigger system needs to maintain an output rate of $100 \mathrm{kHz}$ despite the 10-fold increase in luminosity, and that appears to be impossible to achieve using information from calorimeters and muon detectors only. At present, tracking information is used in the High Level Trigger, achieving a rate reduction of a factor of $\approx 100$ in the muon rate, while a flattening of the Level-1 rate as a function of $\mathrm{p}_{\mathrm{T}}$ threshold is observed, which 
suggests that increasing the threshold will not help, unless tracking information is included. Similar evidence is found for electron, tau, and jet trigger.

\section{Major R\&D activities}

The requirements and constraints mentioned above motivate a number of $R \& D$ activities:

- The need of increased radiation tolerance requires to develop new silicon sensors, exploring different materials and technologies.

- The higher particle density requires higher granularity, which translates to higher channel count; new electronics in the most advanced sub-micron technologies allows to squeeze ten times more channels in the same volume without increasing too much the power budget. However, the lower power per channel is mostly achieved thanks to lower supply voltage, which leaves the problem of limiting the current in the supply cables. This implies that current has to be brought in at higher voltage, which also helps to reduce the mass of the conductors inside the tracking volume.

- Despite the use of more advance electronics technologies, the power dissipated in the detector may be larger than the present one and the required operating temperature for the sensors will be most likely significantly lower (from $-10^{\circ} \mathrm{C}$ to $-20^{\circ} \mathrm{C}$ ), while the coolant temperature cannot be lowered significantly (hard limit at $-30^{\circ} \mathrm{C}$ from cooling channels insulation) and the flow in the detector cannot be significantly increased (size and available number of cooling pipes fixed). That requires a more effective cooling method, providing higher efficiency in the heat removal with reduced mass of pipes, coolant and heat exchangers, which also helps reducing the total amount of material.

- Delivering information for the level-1 trigger involves sending out signals at $40 \mathrm{MHz}$, which requires a strong data reduction to be done in the front-end electronics, in order to keep the overall bandwidth at an acceptable level. The strategy consists in exploiting the strong bending power of the $4 \mathrm{~T}$ magnetic field to design modules that are able to reject in real time signals from low-p particles ("p $\mathrm{p}_{\mathrm{T}}$ modules").

- Due to the complex functionalities, it is likely that $\mathrm{p}_{\mathrm{T}}$ modules (and $\mathrm{p}_{\mathrm{T}}$ layers) will be relatively heavy and power-hungry. It is therefore mandatory to develop also modules with only standard readout functionalities, with granularity adapted to SLHC conditions, and optimized for low material (exploiting advanced electronics and interconnection technologies). The number of $\mathrm{p}_{\mathrm{T}}$ layers and readout layers will be eventually optimized to maximize performance.

- Data links will also profit from advanced electronics technologies to obtain large bandwidth and robust operation in high-radiation environment. High bandwidth is required to serve a more granular detector with the same power plant, and beyond that, to moderate the power dissipated in data links for the $\mathrm{p}_{\mathrm{T}}$ layers.

A few details about status and perspectives of these $R \& D$ activities are given below. 
Silicon sensors CMS has started an extensive R\&D program with HPK. A dedicated wafer has been designed containing a multi-geometry strips and long pixel field, two sets of dedicated test structures for process monitoring, two mini-sensors, diodes and experimental structures to study channel routing. A huge geometrical and technological phase space will be investigated, by studying the following materials, thicknesses and technologies: p-in-n/FZ (100 $\mu \mathrm{m}, 200 \mu \mathrm{m}, 300 \mu \mathrm{m})$, p-in-n/MCz (200 $\mu \mathrm{m}), \mathrm{n}$-in-p/FZ (100 $\mu \mathrm{m}, 200 \mu \mathrm{m}, 300 \mu \mathrm{m}), \mathrm{n}$-in-p/MCz $(200 \mu \mathrm{m}), \mathrm{p}$-in-n/EPI (75 $\mu \mathrm{m}, 100 \mu \mathrm{m})$, n-in-p/EPI $(75 \mu \mathrm{m}, 100 \mu \mathrm{m})$. The n-in-p wafers will be produced in two varieties: $\mathrm{p}$-spray and p-stop. Some n-in-p and p-in-n structures are ordered with an additional double metal layer for routing evaluations. A detailed program of measurements with mixed irradiation (neutrons plus protons) is planned, including Charge Collection Efficiency, strip characterization, channel isolation, breakdown; beam tests are also planned to evaluate tracking efficiency and resolution. The research program will identify the technology choice at least for radii above $10 \div 20 \mathrm{~cm}$, while also addressing issues related to biasing scheme, geometry and routing. More studies for the inner region are ongoing, investigating 3D pixels, diamond pixels, radiation hard CMOS technology and additional $\mathrm{MCz}$ materials.

Powering Scheme CMS has chosen DC-DC converters as baseline technology to bring the current into the detector at higher voltage. A conversion ratio of $\approx 10$ is envisaged. Such option has been preferred compared to serial powering, since system aspects are less complicated. The main conversion step is realized by an inductor-based buck-converter, capable of providing a current of a few Amps, possibly followed by a switched capacitors converter in a two-step system (e.g. to provide two different voltages for the analogue and the digital part). The crucial issue of the R\&D program is the development of a radiation hard ASIC compatible with "high" $(\mathrm{O}(10 \mathrm{~V}))$ voltage. In addition, many system aspects need careful optimization, including noise pick-up from device switching (conductive noise), and from the air-core inductor (radiative noise), integration with front-end electronics and optimization of Power Supply Rejection Ratio (PSRR), minimization of material budget [3].

$\mathrm{CO}_{2}$ cooling Two-phase cooling based on $\mathrm{CO}_{2}$ has been chosen as baseline to replace the present system, which uses liquid $\mathrm{C}_{6} \mathrm{~F}_{14}$. The choice is largely motivated by the positive experience of NIKHEF with the AMS and LHCb VELO detectors. $\mathrm{CO}_{2}$ has excellent thermodynamic properties for micro-channels; the high latent heat and the high heat transfer coefficient will help to achieve the required cooling efficiency, in a lightweight system, with reduced pipe size and manifolds. Low price and environmental compatibility are also strong advantages. Nevertheless, CMS requirements are completely different compared to $\mathrm{LHCb}$, in terms of pipe layout, acceptable pressure range, operating temperature, power load and overall size of the system, which requires a large $R \& D$ program to develop a suitable system. About 10 laboratories are contributing, addressing different aspects in parallel: system aspects in a large re-circulating system, design and test program for the construction of a full-scale system, performance studies for optimization of working parameters ( $\mathrm{T}, \mathrm{P}$, internal pipes dimensions and shape) and analysis of constraints from outer pipes and safety aspects. The developments are initially focusing on the phase I pixel upgrade, with a long-term view on the full upgrade. 
$\mathbf{p}_{\mathrm{T}}$ modules Several options are considered to realize substantial data reduction in the front-end electronics, in order to be able to provide information to the level-1 trigger. One idea, with several proposed implementations, exploits sensors stacked in pairs with a small separation of $1 \div 2 \mathrm{~mm}$, as shown in the left sketch of Fig. 1: the selection of high-p $\mathrm{p}_{\mathrm{T}}$ particles is done by correlating hits from each sensor, keeping as valid signals only those that have similar $\phi$-coordinates. Simulation studies indicate that with such arrangement a rate reduction of a factor of $\approx 20$ is achievable in the radial range $20 \div 40 \mathrm{~cm}$, yielding a manageable data rate. A possible implementation is shown in the left sketch: signals are transferred from the front-end ASICs of one sensor to the other one, through a "substrate" that also carries power and control signals. ASICs are programmed as "transmitters" in one layer, and as "correlators" in the other layer. The design is compatible with current industrial chip packaging technologies. In most proposals, $\mathrm{p}_{\mathrm{T}}$ modules would have a fine segmentation in the $\mathrm{z}$ view $(\approx 2 \mathrm{~mm})$ and would be deployed in the inner part of the Outer Tracker (just outside the pixel volume), in order to provide also some discrimination on the position of the primary vertex for the level-1 trigger.

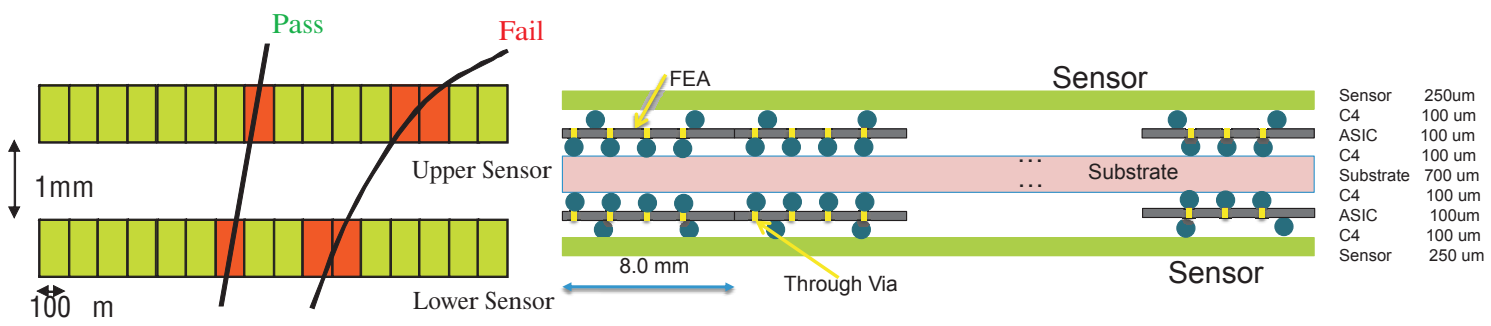

Figure 1: Left: sketch showing the principle of $\mathrm{p}_{\mathrm{T}}$ discrimination in stacked sensor pairs. Right: possible implementation of a $\mathrm{p}_{\mathrm{T}}$ module.

Readout modules For the outer part of the Tracker a readout scheme using binary, unsparsified data transfer has been proposed [4] and the design of a front-end ASIC in $130 \mathrm{~nm}$ CMOS technology is underway. Preliminary studies indicate that the use of advanced interconnection technologies, as well as advanced materials for thermal management, combined with the development of a novel powering scheme and cooling system mentioned above, could allow a large reduction of material compared to the present tracking modules. Three-dimensional models showing a readout module and the arrangement of modules in a larger assembly are shown in Fig. 2.
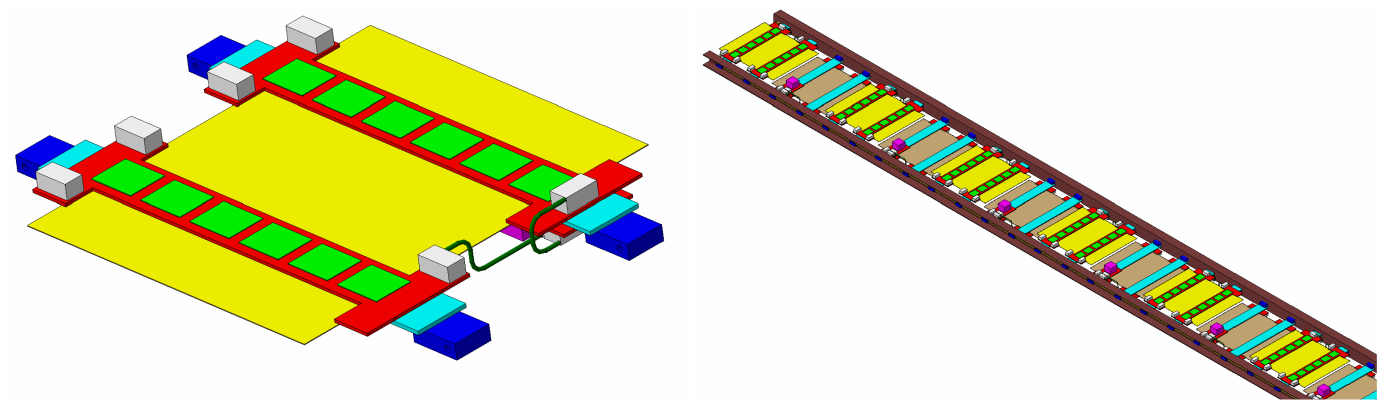

Figure 2: Left: three-dimensional model of a readout module with the associated electronics and support elements. Right: three-dimensional model showing the assembly of modules on larger structures. 
Data links Data transmission is based, at present, on the common projects GigaBit Transceiver [5] and Versatile Optical Link [6]. The chip-set (in $130 \mathrm{~nm}$ ) and the optoelectronic packaging are under development or testing; the development of the front-end interface has started. A first prototype of the full link is expected in about one year. Give the long timescale of the phase II upgrade, it is conceivable that data links could be further developed, taking advantage of even more advanced technologies; the goal would be to improve further the ratio bandwidth/power, which would be beneficial especially for $\mathrm{p}_{\mathrm{T}}$ layers.

\section{Layout studies and conclusion}

The phase-II tracker layout is the focus of studies with the objective to propose a suitable baseline. Monte-Carlo simulations have been developed from the existing code used for simulation of the present tracker. Good progress was made recently and the simulations are now capable of comparing different geometries under SLHC luminosity conditions for different physics channels.

An additional tool has been developed to optimize the layout of the phase-II upgraded tracker. For a chosen set of input parameters (number of layers, module area, splitting between barrel and endcap, etc.) the tool simulates the placement of sensors, mechanics and services throughout the tracker volume in either barrel or endcap (disk) configurations, providing an immediate graphical representation of the layout, along with calculations of the number of modules required, the number of hits per (high $\mathrm{p}_{\mathrm{T}}$ ) track in the range $|\eta|<2.5$ (which is a strong indicator of tracking performance), and the associated costs in terms of the total area of silicon used, the total power consumed, bandwidth and number of readout links. Most importantly, from point of view of final performance, an estimate of the radiation length and interaction length is also provided, following assumptions made on the integration of modules and services.

An example of layout considered is shown in Fig. 3, featuring two $\mathrm{p}_{\mathrm{T}}$ barrel layers outside the pixel volume at radii of about 25 and $35 \mathrm{~cm}$ (pixel detector not shown), surrounded by a four-layer tracker with standard readout functionality. The right plot displays the coverage as a function of pseudo-rapidity:
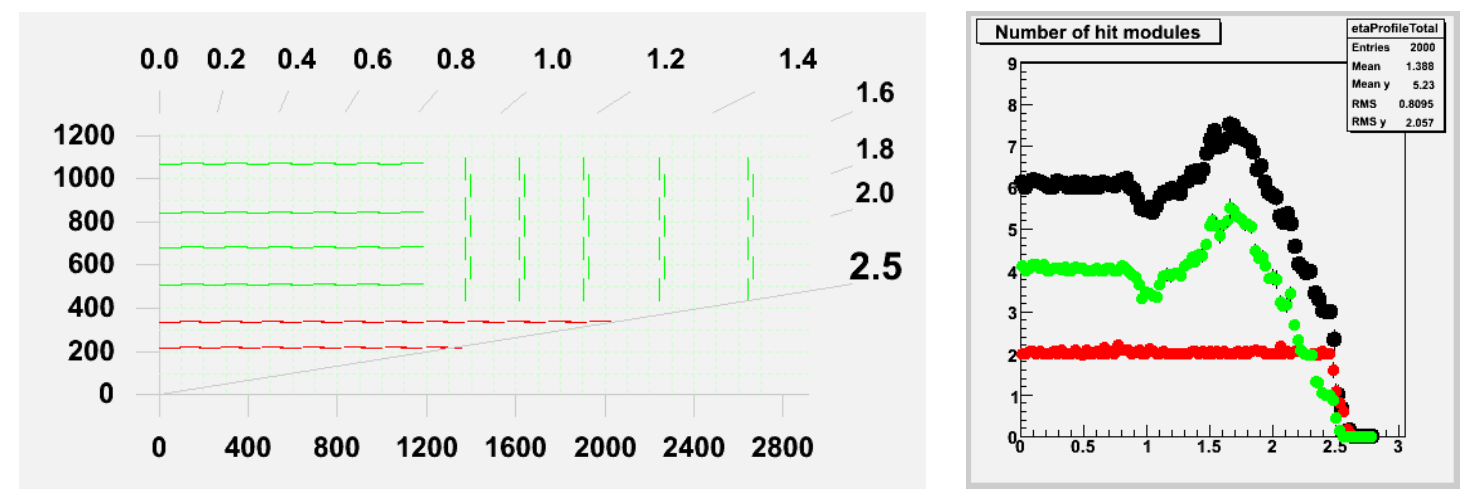

Figure 3: Left: example of one of the phase-II Tracker layouts being studied (pixels not shown): longitudinal quarter-view with axis labeled in mm. Right: geometrical coverage of the layout, providing two coordinates for the level-1 trigger and four additional tracking points (on average) in the full pseudo-rapidity acceptance. 
Using the layout tool described above, a first attempt has been made to estimate the material of such layout, obtaining the results shown in Fig. 4: the left plot shows the radiation length distribution for the outer part only (that has no trigger functionality); the left plot corresponds to the full layout. It is evident that the two stacked $\mathrm{p}_{\mathrm{T}}$ layers dominate the material distribution, due to the intrinsic thickness of the modules (made of two sensors plus a substrate), the higher power dissipation, and the larger data volume that they produce. Although the development of $\mathrm{p}_{\mathrm{T}}$ modules is not yet sufficiently advanced to consider these modeling studies quantitatively reliable, there is clear indication that stacked trigger layers will have a major impact on the material of the tracker, and hence on the tracking performance, and therefore need to be the subject of careful optimization.
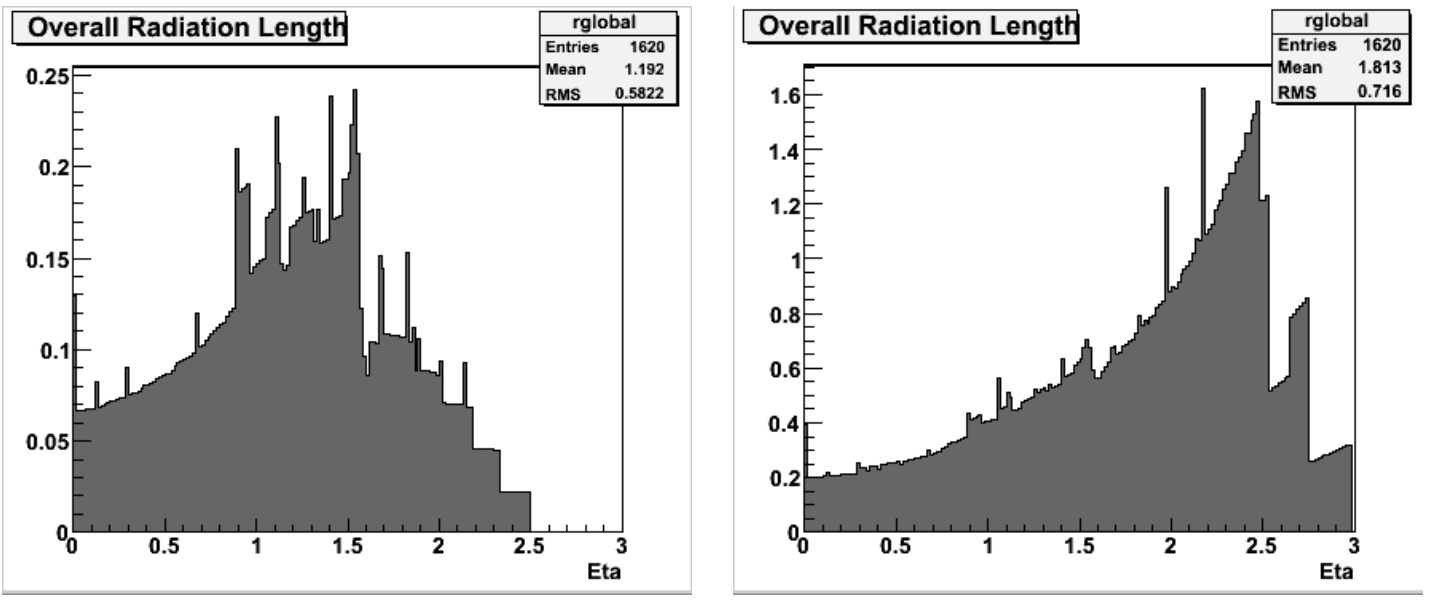

Figure 4: Material modeling studies for the layout shown above. Estimated radiation length as a function of pseudo-rapidity excluding the two $\mathrm{p}_{\mathrm{T}}$ layers (left) and with all layers included (right).

Conclusion A conceptual layout for a Tracker to be operated at SLHC is still under study. The goal of maintaining and possibly improving the present tracking performance in a ten times more dense environment is made particularly challenging by the additional requirement of providing information for the level-1 trigger. $\mathrm{R} \& \mathrm{D}$ is making good progress in all the most challenging areas.

\section{References}

[1] CMS Collaboration, The Compact Muon Solenoid. Technical Proposal, CERN/LHCC 94-38, (1994) CMS Collaboration, Addendum to the CMS Tracker TDR, CERN/LHCC 200-016.

[2] CMS Collaboration, The CMS experiment at the CERN LHC, 2008 JINST 3 S08004.

[3] K. Klein et al., System Tests with DC-DC Converters for the CMS Silicon Strip Tracker at SLHC, Proceedings of the Topical Workshop on Electronics for Particle Physics TWEPP-08.

[4] M. Raymond and G. Hall, CMS microstrip tracker readout at the SLHC, Proceedings of the Topical Workshop on Electronics for Particle Physics TWEPP-08.

[5] P. Moreira et al, The GBT Project, to be published in the Proceedings of the Topical Workshop on Electronics for Particle Physics TWEPP-09.

[6] J. Troska et al., The Versatile Transceiver Proof of Concept, to be published in the Proceedings of the Topical Workshop on Electronics for Particle Physics TWEPP-09. 